J. Appl. Glycosci., 50, 237-240 (2003)

(C) 2003 The Japanese Society of Applied Glycoscience

Proceedings of the International Symposium: New Approaches in Starch Science and Carbohydrate-Active Enzymes

Session I: Structure of Starch and Its Biosynthesis

\title{
Sugar Transporters Involved in Flowering and Grain Development of Rice
}

\author{
(Received September 15, 2002)
}

\author{
Budsaraporn Ngampanya, ${ }^{1,2}$ Taito Takeda, ${ }^{3}$ Jarunya Narangajavana ${ }^{2}$, Akira Ikeda ${ }^{1,4}$ and Junji Yamaguchi ${ }^{1, *}$ \\ 'Division of Biological Sciences, Graduate School of Science, Hokkaido University (N10-W8, Kita-ku, Sapporo 060-0810, Japan) \\ ${ }^{2}$ Department of Biotechnology, Faculty of Science, Mahidol University (Rama 6 Road, Bangkok 10400, Thailand) \\ ${ }^{3}$ Nagoya University Bioscience Center (Chikusa, Nagoya 464-0860, Japan) \\ ${ }^{4}$ CREST, JST (Japan Science and Technology Corporation)
}

\begin{abstract}
Sucrose and hexoses derived from sucrose breakdown are importance for early grain development in cereals. These assimilates were used to promote division and elongation, differentiation of the cells and deposition in storage organs. OSMST5 (Oryza sativa monosaccharide transporter $\underline{5}$ ), OsSUT1 (Oryza sativa sucrose transporter 1 ) and $O S S U T 2$ (Oryza sativa sucrose transporter $\underline{2}$ ) have been cloned from rice. All mRNAs of these functional sugar transporters were detected during flowering and grain filling stage. The OsMST5 and $O S S U T 2$ mRNAs were exclusively detectable before the pollinating stage by Northern analysis and the $O s$ SUT2 mRNA was localized in archespores and pollen mother cells by in situ mRNA detection, indicating that these gene products are involved in import of sucrose to promote pollen development at the early stage. Moreover, the OSSUT1 which is known to be phloem companion cell-specific expression in source organs, was also expressed in the sink organs as well as modified aleurone cells of the developing endosperm. These results indicate that the OSSUTI plays a role in re-uptake of sucrose from endosperm cavity to accumulate starch in grain filling stage.
\end{abstract}

Key words: endosperm, rice, sucrose, sugar transporter

Developing grain is believed to be a good model system for understanding transport and deposition of photosynthetic assimilates in plants. Generally, the event of cell division, maturation and storage emerge in a number of plants. Different seed species are able to deposit the storage product starch, protein and oil in different proportions. According to the filial body (embryo and endosperm) in developing grain is symplastically separated from maternal pericarp and seed coat by the seed apoplast (Fig. 1), it requires post-phloem sugar flow to support growth and development of the filial body. Therefore active transport system is necessary for deliver the compounds. In legumes, sugars in the apoplastic space are imported across the cotyledons by some sugar transporters located on surface of the filial cells. ${ }^{2)}$ In cereals, association with carbohydrate flow in grains of barley and wheat has been reported., ${ }^{3,4}$ Carbohydrates produced by photosynthesis in source organs (green leaves) are flowed through a single or some vascular bundles which cross locating the longitudinal length of grain pericarp ${ }^{4)}$ and are delivered via symplastic pathway to seed apoplast from the vascular bundle(s). At the crease vein area, however, symplastic pathway within maternal cells is protected from a cuticle layer surrounding the inner surface of pericarp. ${ }^{5)}$ In order to supply nutrients for the filial cells, carbohydrates are taken up from seed apoplast by sugar transporters as same as in legume even filial storage cells of grain legume instead of endosperm (Fig. 1). In case of rice, though it is grouped into cereal crop but the pattern of vascular bundle locating on seed grain is different.

\footnotetext{
${ }^{*}$ Corresponding author (Tel. \& Fax. +81-11-706-2737, E-mail:
} jjyama@sci.hokudai.ac.jp).
Vascular bundle of rice seed extends along the length of grain. ${ }^{6}$ Carbohydrates are transported through that route. Nucellar cells which are formed from cuticle layer are thought to be a critical factor for carbohydrate efflux. ${ }^{7.8)}$ The membrane influx of carbohydrates is predicted on modified aleurone cells ${ }^{7)}$ (Fig. 1).

In addition the pollen grain has no plasmodesmata which are intercellular connection to the sporophytic tissue and it dependents on an external supply of nutrients. Import of carbon and nitrogen is necessary for development of the pollen grain in anthers. Pollen thus seems to be an ideal subject in which to study the role of transport in nutrition and development. In developing pollen grain, strong sinks required carbohydrates for maturation, germination and growth ${ }^{9)}$ and monosaccharide transporter seems to play a pivotal role involved in this physiological task. Pmt1 and AtSTP2, monosaccharide transporter in petunia and Arabidopsis, respectively, are activated after the pollen mitosis and involved in pollen tube growing. ${ }^{10,11)} \mathrm{Be}-$ side these monosaccharide transporters, there have been detected of AtSUC1 (sucrose transporter) mRNA and protein in parenchymatic cells surrounding the vascular bundle of anther connective tissues before the dehiscence of anther. This transporter is believed to be a factor for pollen tube germination.

From previous study ${ }^{1)}$ we cloned OSMST5 and OSSUT2 which are involving in pollen development. In the present report we performed in situ hybridization to explain the role of sugar transporters in developing grain.

\section{MATERIALS AND METHODS}

Materials. Rice seeds (Oryza sativa L.; Japonica-type 


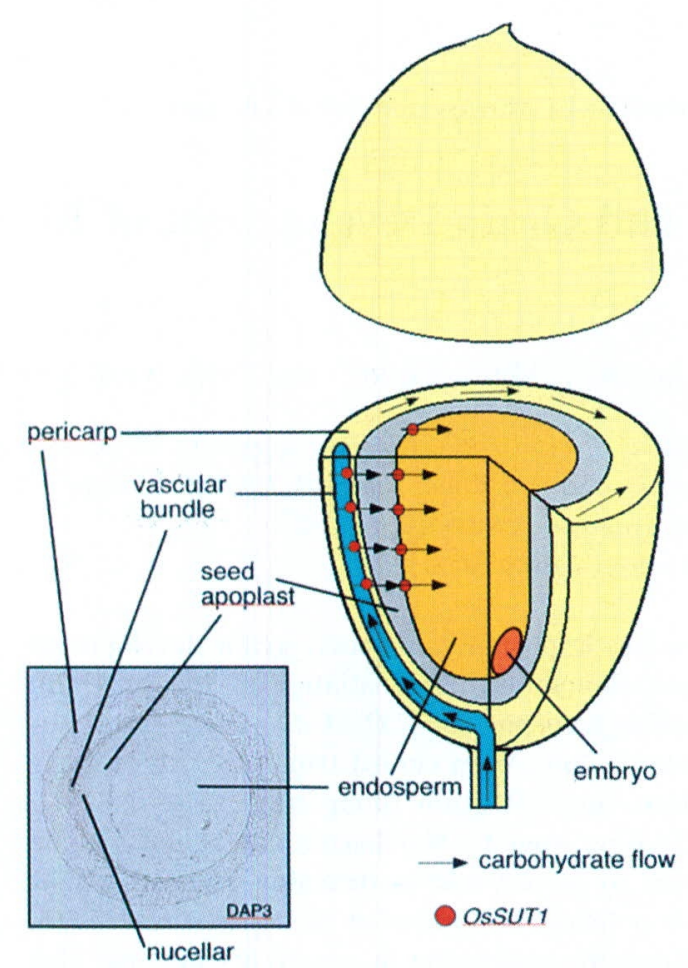

Fig. 1. Localization of OsSUT1 mRNA in vascular bundle of developing seed (Left) and schematic of carbohydrate flow in rice seed.

OSSUT1 antisense probe was used to perform in situ hybridization.
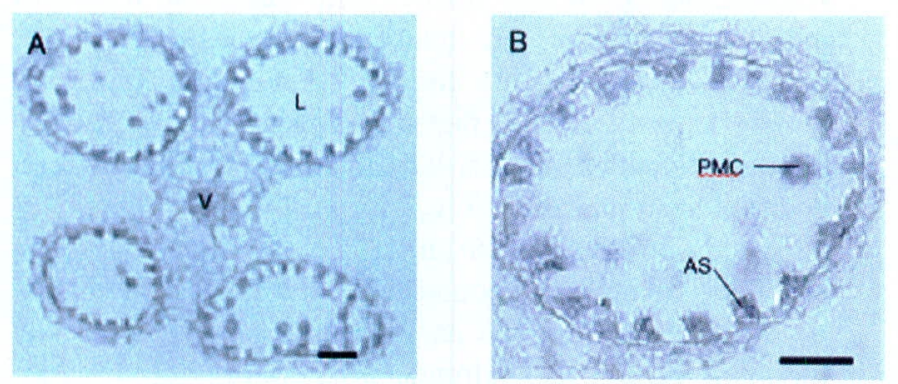

C

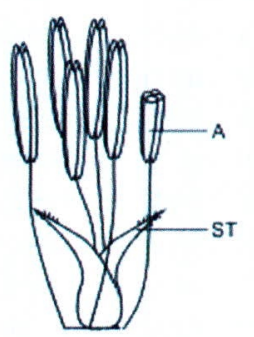

Exterior

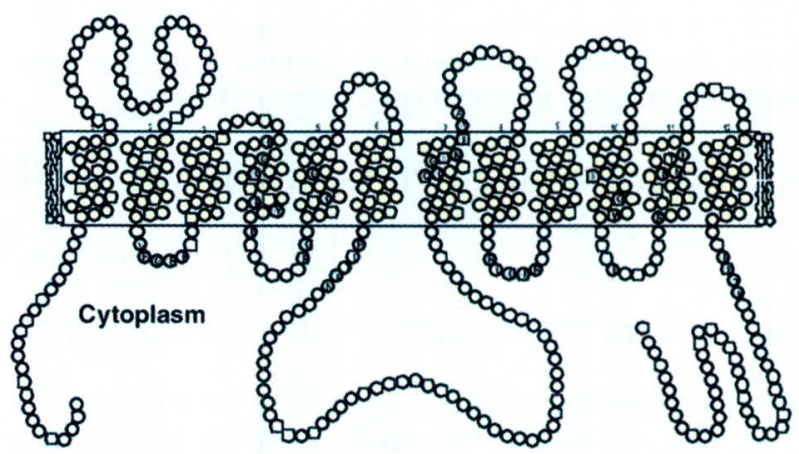

Fig. 2. A membrane-spanning model of OSMST5.

Schematic representation of a twelve-transmembrane spanning model for OsMST5. Letters inside the circles indicate the amino acid residues composing the motifs of the monosaccharide transport, and shaded circles indicate the conserved amino acid residues in the OsMST5 protein.

Fig. 3. Localization of OsSUT2 mRNA in anther (A, B, D).

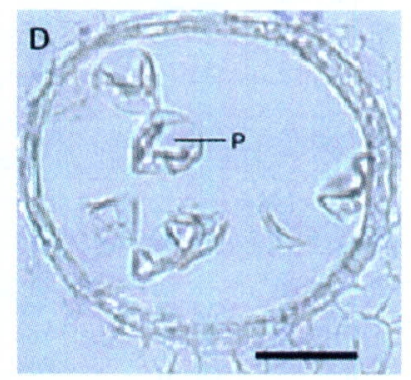

OSSUT2 antisense probe was used to perform in situ hybridization. A, B and D, cross section of anther; C, rice flower. A, anther; AS, archespore; L, loculus; P, pollen; PMS, pollen mother cell; ST, stigma; V, vascular bundle. Bar $=0.1 \mathrm{~mm}$.

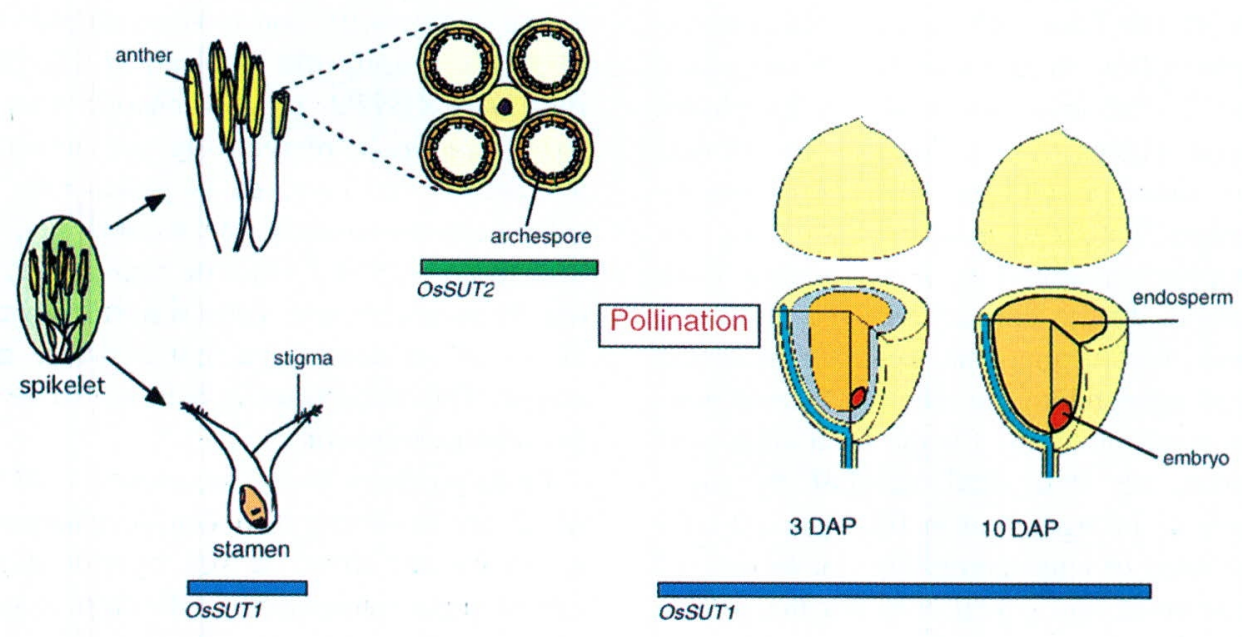

OSMST5?

Fig. 4. Sugar transporters during flowering and grain development in rice.

Line indicates the period for the gene expression of specific-sugar transporter gene. 
cultivar: Nipponbare) were sown and grown in artificial soil in a greenhouse. Plants at flowering and grain maturating stage were used as materials for the experiments.

Cloning of sugar transporter. OSMST5 and OSSUT2 cDNAs sequence were obtained from the Rice Genome Research Program. ${ }^{1)}$ The ABI 373A DNA sequencer (Perkin-Elmer Co., NJ, USA) was used to examine the cDNA sequences by means of dideoxy-chain termination. Sequence alignment of OSSUT2 is identical to that of OsSUT3 (accession no. AB071809 by Hirose et al.).

Characterization of monosaccharide transporter. To predict transmembrane helices in OsMST5, the topological pattern of the deduced amino acid sequence was performed by TMHMM (V.2.0). ${ }^{12,13)}$ For sugar uptake assay, D-xylose was used as a transport substrate to analyze transport activity and for estimating the energy-dependent active transport system of OsMST5.

In situ hybridization of OsSUT1 and OsSUT2. Rice panicle at indicating date during flowering and grain filling stage was harvested and fixed in FAA solution to perform in situ hybridization by using OsSUT1 and OsSUT2 antisense cDNAs as probes. ${ }^{15)}$

\section{RESULTS AND DISCUSSION}

\section{Characterization of monosaccharide transporter, OsMST5.}

Although three distinct monosaccharide transporters $(O S M S T s 1-3)$ in rice have been reported by Toyofuku et al. ${ }^{16)}$ there have not been any reports of those transcripts detectable in the developing grain. To study on the role of sugar transporters during grain development, the new functional monosaccharide transporter cDNA OsMST5, which transcript is detectable at the flowering stage, was cloned and characterized. Analysis of the deduced amino acid sequence by TMHMM (V.2.0 $)^{12,13)}$ revealed the transmembrane helices of OsMST5 (data not shown). Two sets of putative transmembrane domains separated by a central long hydrophilic region (Fig. 2) were observed. Therefore, it is possibly to suggest that OsMST5 is a membranespanning protein with 12 transmembrane regions (Fig. 2) and is consistent with those of sugar transporters in microbes, mammals, and plants. To analyze the function of OsMST5, cDNA was subcloned in a $G A L$ expression vector and transformed into LBY416, a strain of $S$. cerevisiae in which high-affinity glucose transport activity is kept low according to three monosaccharide transporter-related genes, $H X T 2$, GAL2 and $S N F 3$, have been interrupt. ${ }^{16)}$ GAL2 promoter allowing the expression occurs in the presence of galactose was used. The transport activity of the subcloned cDNA was compared with other monosaccharide transporter in rice, OsMST3, since it had the intense activity in comparison with OsMST1 and OsMST $2 .{ }^{16)}$ The glucose transport activity of OsDMST5 was approximately three times lower than OsMST3 activity and nearly two times higher than empty vector, pTV3e activity (data not shown). For further study on the energydependent transport activity of OsMST5, D-xylose was used. LBY416 yeast cells harboring OsMST5 could uptake D-xylose rapidly when ethanol was added. Whereas uncoupler CCCP declined this energization on the plasma membrane of yeast cells (data not shown). These data are consistent with a proton-coupled uptake mechanism. The energy generating by ethanol allowed high rate uptake of $\mathrm{D}$-xylose into cells and then rapidly metabolized. According to the activity of CCCP as a plasma membrane deenergization, the transport of $\mathrm{D}$-xylose was rapidly decreased. These results indicate that the OsMST5 serves as an energy-dependent monosaccharide transport system, possibly a $\mathrm{H}^{+}$-symporter.

\section{OsSUT1 and OSSUT2 mRNA localization.}

To study the role of sucrose transporter in developing seeds, in situ hybridization by using antisense of $O S S U T 1^{14)}$ and $O S S U T 2^{1)}$ as probes was performed. The OSSUT1 mRNA was restrictly detectable in phloem companion cells in the leaf sheath ${ }^{15)}$ and in the most outercell layer (transfer cells/modified aleurone cells in cereals) of the developing grain (see lower left panel of Fig. 1). Moreover mRNA of OsSUT2 was detectable in developing pollen (Fig. 3). These results in present studies demonstrates that sucrose transporter is not only importance for delivering sugar in source organ but also plays critical roles in sink organs as well as developing grains and pollen grains.

\section{Role of sugar transporter in developing seed.}

As the route of carbohydrate flow in rice grain extends along the length of grain, therefore the mechanisms of sugar transport from maternal cells into fillial cells is likely distinguish from previous reports in legume. Although there has been studied in the role of sugar import and metabolism in barley as monocotyledonous species, ${ }^{17)}$ morphological structures between barley and rice grains are distinct. From all data in this study it might be concluded that as shown in Fig. 4: 1) at the early stage of pollen development, sucrose unloaded from vascular bundles of anther filament was imported into archespores and pollen mother cells by OsSUT2 afterward probably OsMST5 function as an importer of cell wall-bouhd invertases-mediate derivatives to support the development of pollen grain in anther; 2) furthermore OsSUT1 expressed in transfer/modified aleurone cells in the developing endosperm plays a role in re-uptake of sucrose from endosperm cavity to accumulate starch in grain filling stage. The regulations during the import are open to dissolve by further investigations.

This work has been supported by Research for the Future (JSPS00L01603) from the JSPS (Japan Society for the Promotion of Sciences) and CREST of the JST (Japan Science and Technology). BN acknowledges a UDC scholarship supported by The Ministry of University Affair, Thailand.

\section{REFERENCES}

1) T. Takeda, K. Toyofuku, C. Matsukura and J. Yamaguchi: Sugar transporters involved in flowering and grain development of rice. J. Plant Physiol., 158, 465-470 (2001).

2 ) H. Weber, L. Borisjuk and U. Wobus: Sugar import and metabolism during seed development. Trends Plant Sci., 2, 169174 (1997).

3 ) H. Cook and K.J. Oparka: Movement of fluorescein into iso- 
lated caryopses of wheat and barley. Plant Cell Env., 6, 239$242(1983)$.

4 ) F.A.K. Sakri and J.C. Shannon: Movement of ${ }^{14} \mathrm{C}$-labeled sugars into kernals of wheat (Triticum aestivum L.). Plant Physiol., 55, 881-889 (1975).

5 ) S.Y. Zee and T.P. O'Brien: Studies on the ontology of the pigment strand in the caryopsis of wheat. Aust. J. Biol. Sci., 23, 1153-1171 (1970).

6 ) S.Y. Zee: Vascular tissue and transfer cell distribution in the rice spikelet. Aust. J. Biol. Sci., 25, 411-414 (1972).

7 ) K.J. Oparka and P. Gates: Transport of assimilates in the developing caryopsis of rice (Oryza sativa L.): Ultrastructure of the pericarp vascular bundle and its connections with the aleurone layer. Planta, 151, 561-573 (1981).

8 ) K.J. Oparka and P. Gates: Transport of assimilates in the developing caryopsis of rice (Oryza sativa L.): The pathway of water and assimilated carbon. Planta, 152, 388-396 (1981).

9 ) L.E. Williams, R. Lemoine and N. Sauer: Sugar transports in higher plants: A diversity of roles and complex regulation. Trends Plant Sci., 5, 283-290 (2000).

10) B. Ylstra, D. Garrido, J. Busscher and A.J. van Tunen: Hexose transport in growing petunia pollen tubes and characterization of a pollen-specific, putative monosaccharide transporter. Plant Physiol., 118, 297-304.

11) E. Truernit, R. Stadler, K. Baier and N. Sauer: A male gametophyte-specific monosaccharide transporter in Arabidopsis. Plant J., 18, 191-201 (1999).
12) A. Krogh, B. Larsson, G. von Heijine and E.L.L. Sonnhammer: Predicting transmembrane protein topology with a hidden Markov model: Application to complete genomes. J. Mol. Biol., 305, 567-580 (2001).

13) E.L.L. Sonnhammer, G. von Heijine and A. Krogh: A hidden Markov model for predicting transmembrane helices in protein sequences. in Proceeding of the Sixth International Conference on Intelligent Systems for Molecular Biology. J. Glasgow, T. Littlejohn, F. Major, R. Lathrop, D. Sankoff and C. Sensen, eds., AAAI Press, Menlo Park, CA, pp. 175-182 (1998).

14) T. Hirose, N. Imaizumi, G.N. Scofied, R. Furbank and R. Ohsugi: cDNA cloning and tissue specific expression of a gene for sucrose transport from rice (Oryza sativa L.). Plant Cell Physiol., 38, 1389-1396 (1997).

15) C. Matsukura, T. Saitoh, T. Hirose, P. Perata and J. Yamaguchi: Sugar uptake and transport in rice embryo: Expresssion of companion cell-specific sucrose transporter (OsSUT1) induced by sugar as well as light. Plant Physiol., 124, 85-94 (2000).

16) K. Toyofuku, M. Kasahara and J. Yamaguchi: Characterization and expression of monosaccharide transporters (OSMSTS) in rice. Plant Cell Physiol., 41, 940-947 (2000).

17) W. Weschke, R. Panitz, N. Sauer, Q. Wang, B. Neubohn, H. Weber and U. Wobus: Sucrose transport into barley seeds: Molecular characterization of two transporters and implications for seed development and starch accumulation. Plant J., 21, 455-467 (2000). 